\title{
Predication Versus Membership in the Distinction Between Logic as Language and Logic as Calculus
}

\author{
Nino B. Cocchiarella
}

\begin{abstract}
Two types of framework are distinguished regarding the nature of logic and the logical analysis of natural language. In the first, logic is a calculus subject to varying set-theoretic interpretations over domains of varying cardinality, and in this sense is based on a theory of membership in a set. This type need not restrict its analyses of natural language to extensional discourse only; e.g., Richard Montague's sense-denotation intensional logic, which has been used to provide analyses of intensional discourse, is really a type-theoretical set theory supplemented with a theory of senses. The analyses this type of framework provides are not entirely satisfactory, however, for reasons related to the way that intensional entities are analyzed in terms of membership in a set. The second type of framework, where logical forms are semantic structures in their own right, is based on predication as described in a formal theory of universals. This type of framework: gives a more adequate analysis of natural language and can be developed in a type-free way without generating the logical antinomies. Also, because a set-theoretic semantics provides only an extrinsic characterization of validity for this type of framework, such a semantics cannot be used to show that the laws of logic of this type of framework must be essentially incomplete.
\end{abstract}

There are two major doctrines regarding the nature of logic today. The first is the view of logic as the laws of valid deductive inference, i.e., logic as calculus. This view began with Aristotle's theory of the syllogism, or syllogistic logic, and in time evolved first into Boole's algebra of logic and then into quantificational logic. On this view, logic is an abstract calculus capable 
of various interpretations over domains of varying cardinality. Because these interpretations are given in terms of a set-theoretic semantics where one can vary the universe at will and consider the effect this has on the validity of formulas, this view is sometimes described as the set-theoretic approach to logic (see van Heijenoort 1967, p. 327).

The second view of logic does not eschew set-theoretic semantics, and it may in fact utilize such a semantics as a guide in the determination of validity. But to use such a semantics as a guide, on this view, is not the same as to take that semantics as an essential characterization of validity. Indeed, unlike the view of logic as calculus, this view of logic rejects the claim that a set-theoretic definition of validity has anything other than an extrinsic significance that may be exploited for certain purposes (such as proving a completeness theorem). Instead, on this view, logic has content in its own right and validity is determined by what are called the laws of logic, which may be stated either as principles or as rules. Because one of the goals of this view is a specification of the basic laws of logic from which the others may be derived, this view is sometimes called the axiomatic approach to logic.

There is no uniformity among the advocates of either view of logic as to what theory of logical form should be taken as a definitive system of logic. First-order logic, for example, can be the favored system for an advocate of either view; but then so might a form of higher-order logic as well. Even the distinction between intensional and extensional logic, as we will see, fails to mark a clear line between the two views. A set-theoretic semantics for intensional logic is also called possible-worlds semantics. In this paper we will be concerned only with one or another version of intensional logic as a way of explaining the distinction between these two views of logic.

The main problem for the axiomatic approach is whether or not there can be a complete, recursive axiomatization of the laws of logic of a given theory of logical form. When the answer is affirmative, a set-theoretic semantics may help in establishing that fact; but in that case, on the view of logic in question, the completeness theorem we establish in terms of such a settheoretic semantics "is deprived of sense ... by the very use we make of it. It is a case, in Wittgenstein's figure, of kicking away the ladder by which we have climbed" (Quine 1968, p. 297). The possibility of a negative answer, however, is another matter altogether, and it is one of the problems we shall consider in this paper.

The historical antecedent of the second view of logic, incidentally, was 
Leibniz's somewhat visionary goal of a lingua philosophica or characteristica universalis. For Frege this goal meant the construction of "a logically perfect language" that was not only complete with respect to the laws of valid inference but which also would enable us to give a conceptual or logical analysis of the different possible statements we might make and to which such laws might apply. Instead of logic being an abstract calculus subject to varying interpretations, logic on this view has content of its own and is a language in its own right. That is why this approach is sometimes described as the view of logic as language (see van Heijenoort 1967, pp. 324-30).

This is not to say that all content on this view of logic is logical content, i.e., content expressible in purely logical terms. Rather, the nonlogical content that can be expressed in a such a logically perfect language is really content that can be expressed in an applied form of the theory of logical form characterizing the logico-grammatical structure of that language. In this regard, the language in the idea of logic as language is not intended as a substitute for natural language so much as a way of providing a semantics for natural language. Applied logical forms, in other words, are really semantic structures in their own right, and it is by assigning such logical forms to the expressions of a natural language that one provides an interpretation or semantics for those expressions. Such an assignment amounts, on this view, to a conceptual or logical analysis of those expressions. That is why an advocate of this view might well consider an applied theory of logical form as a lingua philosophica.

In contrast, applied logical forms on the view of logic as calculus have no more semantic content of their own than do unapplied logical forms. Both are merely expressions of an abstract calculus, and any content they might otherwise be taken as having is a matter of an external set-theoretical semantics. In this regard, the real contrast between logic as calculus and logic as language is more appropriately seen as a contrast between the external set-theoretical semantics underlying an applied system of logic as an abstract calculus and the internal semantics expressed in the logical forms of that system as a language in its own right. This contrast, I believe, can ultimately be best explained in terms of the difference between a theory of membership in a set on the one hand and a theory of predication on the other.

On the view of logic as language, the most fundamental of all logical forms are the forms of predication, since these are the forms that underlie any possible assertion that we might make in language. Indeed, so fundamental are these forms on the view in question that different theories of logical form as 
alternative accounts of logic as language are for the most part really based on alternative theories of predication. In the history of philosophy, these alternative theories have generally been presented as theories of universals, the three most prominant being nominalism, conceptualism and realism. This is because on each of these theories a universal is that which can be predicated of things, and in fact it is the predicable nature of a universal that constitutes its universality. Sets, it should be noted, are not universals in this sense, though of course they are abstract entities; but being an abstract entity is not the same as being a universal.

We will not go into the formal differences between different theories of universals here, having already covered that ground in my 1986 book and my 1988 article. Nevertheless, we do note that insofar as a theory of logical form is based on a formal theory of predication, which in turn is associated with a theory of universals, then, on the logic as language view of logical forms as semantic structures in their own right, we may take that theory of logical form as being itself the explanation that its associated theory of universals purports to give of the predicable nature of universals, i.e., of that in which the universality of universals consists. In this regard, we maintain in what follows that what distinguishes the view of logic as language from that of logic as calculus is that on the former logic is based on a formal theory of predication, whereas on the latter it is based, at least semantically, on membership in a set. This is because on the latter view validity is characterized essentially in terms of a set-theoretic semantics, whereas on the view of logic as language validity is a matter of the laws of logic that are expressed in terms of the theory of logical form in question. This difference, as we shall see, is not unrelated to the problem of the possible incompleteness of a given theory of logical form.

On the set-theoretic approach, predication is interpreted in terms of membership, and not membership in terms of predication. Predication, in other words, has no real significance on this approach over and above its analysis in terms of membership in a set. This is true, moreover, not just for the forms of predication of a logical calculus but for those of natural language as well. That is, one can give a set-theoretic semantics for a recursively specified natural language just as one can for a logical calculus. On the set-theoretic approach, accordingly, there is no need for a theory of logical form as an explanation of the predicable nature of universals, because predication on this approach is ultimately to be analyzed in terms of membership. This suggests that set theory can itself be taken as a lingua philosophica or frame- 
work within which philosophical analyses can be given; and indeed that is the point of our claim that the real contrast between logic as calculus and logic as language is between a theory of membership in a set and a theory of predication. The problem here, however, is whether any analysis of predication in terms of membership is really adequate, or whether there really is something to the idea of logical forms, especially those regarding predication, as semantic structures underlying the expressions of natural language.

\section{The Problem with a Set-Theoretic Semantics of Natural Language}

A good example of the supposed superfluous role of logical forms as semantic structures of the sentences of natural language is Montague's 'English as a Formal Language' [EFL]. Here Montague uses set theory to construct the syntax and semantics of a fragment of English in a way that resembles the construction in set theory of the syntax and semantics of a first-order modal predicate calculus. For example, syntactic categories are recursively defined for this fragment of English in terms of certain basic expressions of those categories and a system of grammatical rules that generates complex expressions from simpler ones. An expression that belongs to any one of these categories is a meaningful expression of English, and as such it is called a "denoting" expression of its respective category. The meaning of such an expression in any model for the fragment, in other words, will be the denotation it is assigned in such a model (relative to an assignment of values to variables).

A model in Montague's semantics for his fragment of English is a settheoretic structure consisting of what we are to think of as a set of possible individuals, a set of possible worlds, and certain functions defined on these sets assigning appropriate types of denotations (relative to an assignment of values to the variables) to the basic expressions of the syntactic categories of that fragment. Of course, the goal of the semantics is to assign denotations to all of the meaningful expressions, and not just to the basic ones. But unlike the situation in the predicate calculus, some denoting expressions are ambiguous in that they can be built up from the basic ones in several different ways on the basis of the grammatical rules. This is as it should be, of course, because the point is to formalize a fragment of a natural language where such 
ambiguity is common. Montague overcomes this problem by first associating a tree-structure with a denoting expression that provides an analysis of how that expression is generated. Ambiguous expressions will then be assigned more than one tree-structure. The denotation of a denoting expression will be determined then not just relative to a model and an assignment of values to variables, but to a tree-structure analysis of that expression as well. The result is a semantical analysis within set theory of both ambiguity and the relation of logical consequence for the fragment of English in question.

Now there are certain important assumptions in Montague's approach in 'English as a Formal Language' that may be challenged. One such assumption, for example, is that "the construction of syntax and semantics must proceed hand in hand" ([EFL], p. 210) and that the basic goal of serious syntax and semantics is the construction of a theory of truth (see Moravcsik 1979, pp. 3-15, for a criticism of this assumption). Another is Montague's use of Frege's principle of compositionality, e.g., that truth-values are to be assigned to sentences by assigning extra-linguistic entities to all of the expressions involved in the generation of those sentences, and in such a way that "the assignment to a compound will be a function of the entities assigned to its components" ([EFL], p. 217; see Hintikka 1980 and 1981, section 6, for criticism of this assumption). We will not take up these assumptions here ourselves, however, but will instead consider Montague's use of set theory as a theoretical framework in which to analyze such intensional entities as propositions, properties and relations in intension as these are normally expressed in natural language. For the idea that such analyses can be given amounts in effect to the claim that set theory can be used as a lingua philosophica; and indeed Montague was at one time quite clear that that was in fact his goal.

Philosophy, on this view, has "as its proper theoretical framework set theory with individuals and the possible addition of empirical predicates" ("On the Nature of Certain Philosophical Entities' [PE], p. 154). Philosophical analyses, in other words, are to be carried out as definitional extensions of set theory, supplemented when needed with empirical predicates and individuals as concrete urelements. In particular, Montague's formalization of the syntax and semantics of a fragment of English is really an analysis carried out by means of a definitional extension of set theory supplemented with the notions of a possible individual and a possible world. On this analysis, where $A$ is the set of possible individuals and $I$ is the set of possible worlds of a model, the set of propositions or possible denotations of formulas is defined 
as the set $U_{1}=\{0,1\}^{I}$, i.e., as the set of functions from possible worlds to truth-values (where 0 represents falsehood and 1 represents truth). Similarly, the set of properties or possible denotations of one-place predicates and the set of two-place relations-in-intension are defined as the sets $U_{2}=U_{1}^{A}$ and $U_{3}=U_{1}^{A \times A}$, respectively.

Now the question that arises here is how seriously are we to take these definitions? In particular, do propositions, properties and relations in intension really have the kind of dependence on the sets of possible individuals and possible worlds they are assumed to have on Montague's analysis? One consequence of this analysis, for example, is that properties and relations-inintension are identical if they have the same extension in every possible world (of a given model), and similarly, that propositions are identical if they have the same truthvalue in every possible world. This means, as Montague himself noted, "that if $\varphi$ and $\chi$ are logically equivalent sentences (with respect to given analyses $f$ and $g$ ), then 'John believes that $\varphi$ ' and 'John believes that $\chi$ ' will turn out also to be logically equivalent (with respect to analyses that contain $f$ and $g$ as parts)" ([EFL], p. 218). This leads to what Hintikka has called the problem of logical omniscience, i.e., the problem of believing or knowing all of the logical consequences of what one believes or knows, respectively.

We can avoid this consequence, as Montague does in 'Universal Grammar' [UG], by distinguishing between designated and undesignated possible worlds (of a given model), where in the latter logical constants can be given nonstandard interpretations. The logical equivalence of two expressions will then depend only on the extensions they have in designated worlds, while synonymy will depend on the extensions they have in the nondesignated worlds as well (cf. [UG], p. 231). Of course this assumes that some account can be given in purely set-theoretical terms of the distinction between designated and undesignated possible worlds (or of designated and undesignated contexts of use within possible worlds).

But even aside from assuming a new and unexplained distinction between designated and undesignated possible worlds there still remains the problem of the individuation of propositions, properties and relations-in-intension in terms of the sets of possible individuals and possible worlds of a given model. For this means that within set theory as a purported lingua philosophica it is meaningless to talk about the same proposition, property or relation-inintension across models that differ, however trivially, in their sets of possible individuals or possible worlds. Certainly, this sort of dependence is contrary 
to the way intensional entities are represented in natural language. In particular, just as properties are not individuated in terms of their instances, they are also not ordinarily thought of as being individuated in terms of the instances they have in different possible worlds, designated or undesignated; and the idea that we cannot meaningfully speak of the same property as being represented in different models indicates that something is radically wrong with a set-theoretical analysis of properties.

Of course, much depends here on how we are to understand the notions of a possible world and a possible individual. Montague himself only asked that we think of two of the constituents of a model as the sets of possible individuals and possible worlds of that model (cf. [EFL], p. 192); but in doing so he implicitly assumed that we understand the notions of a possible world and possible individual. In order to be articulated, however, such an understanding would seem to presuppose a modal or intensional logic either as an adjunct to set theory itself or as a superseding theory of predication in which membership is no longer a fundamental or primitive concept. In either case, set theory, or a theory of membership alone, will not suffice as a theoretical framework for philosophy, or at least not for a philosophy that purports to provide a semantics for natural language. Whatever its merits as a mathesis universalis, set theory, or a theory of membership alone, does not suffice as a lingua philosophica.

\section{Intensional Logic as a New Theoretical Framework for Philosophy}

Montague did not himself remain satisfied with set theory as a lingua philosophica, and in the end he proposed instead the construction of an intensional logic as a new theoretical framework within which to carry out philosophical analyses. Thus, according to Montague, "philosophy is always capable of enlarging itself; that is, by metamathematical or model-theoretic meansmeans available within set theory - one can 'justify' a language or theory that transcends set theory, and then proceed to transact a new branch of philosophy within the new language. It is now time to take such a step and to lay the foundations of intensional languages" ([PE), p. 155).

Actually Montague went on to construct not one but two intensional logics, but only the first is a theory of logical form in the traditional sense of 
being based on predication. This was Montague's higher-order modal predicate logic, which he originally described as a second-order predicate logic with third-order predicate constants, but which can be easily extended to include predicate expressions of all finite orders (as is done in Gallin (1975, chap. 3), and as we will assume it to be here). Montague's second intensional logic, on the other hand, is really a type-theoretical set theory where sets are represented by their characteristic functions, but extended so as to include a theory of senses as well. That is, where $F$ is the characteristic function of a set $A$, i.e., a function from objects to truth-values (members of $\{0,1\}$ ), then $F(x)=1$, which in Montague's second intensional logic is written as $F(x)$, means the same thing as $x \in A$. Thus, instead of being based on predication, Montague's second intensional logic is really based on membership (as represented by the characteristic function of a set). The distinction may seem unimportant (and we will not be concerned with it in this section), but, in fact, as we shall argue later, it goes to the very heart of the matter of the difference between logic as calculus and logic as language. For now, we want only to claim that the difference between logic as calculus and logic as language applies as much to intensional logic as it does to extensional logic.

Montague's own reason for dropping his first intensional logic in favor of the second had nothing to do with the difference between logic as calculus and logic as language. Rather, it was a result of the apparent need in the application of his first intensional logic to resort to circumlocution and paraphrase in the analysis of the intensional verbs of natural language. In the application of his second intensional logic, in other words, Montague was able to describe a precise translation function that provided a direct analysis of intensional verbs, i.e., an analysis that does not resort to circumlocution and paraphrase. (This may be seen as an argument in favor of the set-theoretical approach, at least when the latter is extended to include a theory of senses; for that, as we have said, is what Montague's second intensional logic really amounts to.)

The problem of translation into a theory of logical form seems to be better appreciated by linguists than by philosophers. For unlike a set-theoretical semantics where recursive or effective truth-conditions are given (as translation rules into set theory) corresponding to the recursive construction of the syntactic categories of a natural language, the assignment of logical forms is generally presented as an intuitive process (or as an art that we learn in elementary logic courses) that depends essentially on circumlocution and paraphrase. Montague, as noted, relied heavily on such circumlocution in 
the philosophical analyses he gave in his first intensional logic. For example, in giving an analysis of

Jones sees a unicorn having the same height as a table actually before him.

that does not involve assuming the existence of sense-data (as some philosophers claim it must), Montague distinguished between 'sees' in its veridical sense and 'sees' in its nonveridical sense. The latter was paraphrased by Montague as 'seems to see', where 'seems' was analyzed as a three-place predicate, ' $x$ seems to $y$ to $F$ '. The phrase 'to $F$ ' is taken in this analysis as an infinitive or nominalized predicate occurring as an abstract singular term. Using $\lambda$-abstracts to represent properties and relations, and as translations of infinitives when occurring in argument positions (where ' $[\lambda z \varphi]$ 'is read 'to be a $z$ such that $\varphi^{\prime}$ ), we can describe Montague's analysis of the above sentence as follows:

$$
\begin{aligned}
& (\exists x)[\text { Table }(x) \wedge \text { Before }(x, \text { Jones }) \wedge \text { Seems }(\text { Jones, Jones } \\
& [\lambda z(\exists y)(\operatorname{Unicorn}(y) \wedge \operatorname{Sees}(z, y) \wedge \text { Has-the-same-height-as }(y, x)])] .
\end{aligned}
$$

No sense-data are involved in this analysis, and, according to Montague, an argument for the existence of sense-data based on the content of the sentence in question must therefore fail (cf. [PE], p. 171).

As another example of the apparent need for circumlocution, consider the following two arguments, one of which is clearly valid while the other is clearly invalid.

Jones finds a unicorn; therefore, there is a unicorn.

Jones seeks a unicorn; therefore, there is a unicorn.

The puzzle here is to explain how of two arguments of apparently the same logical form one can be valid and the other invalid.

Montague's approach in this example was to regard 'tries to find' as a circumlocution for 'seeks', where the verb 'tries' is analyzed as a two-place predicate, ' $x$ tries to $F$ '. Again, the phrase 'to $F$ ' in this analysis represents an infinitive or nominalized predicate occurring as an abstract singular term. Thus, whereas the first argument is assigned the following logical form,

$$
(\exists y)[\operatorname{Unicorn}(y) \wedge \text { Finds }(\text { Jones, } y)] ; \text { therefore, }(\exists y) \operatorname{Unicorn}(y) \text {, }
$$


which is clearly valid, the second argument is assigned the different logical form,

$$
\begin{aligned}
& \text { Tries }(\text { Jones, }[\lambda z(\exists y)(\operatorname{Unicorn}(y) \wedge \text { Finds }(z, y))]) \text {; } \\
& \text { therefore, }(\exists y) \operatorname{Unicorn}(y),
\end{aligned}
$$

which is not valid. In other words, through circumlocution and paraphrase, the two arguments can be seen to have different logical forms after all; and therefore the validity of the one argument need not carry over to the other.

These analyses, it should be emphasized, occur in Montague's application of his first intensional logic, which is really a theory of logical form that is based on predication. Despite the philosophical usefulness of these analyses, however, Montague subsequently came not to depend on them. In particular, Montague became dissatisfied with the use of circumlocution and paraphrase, and he came to maintain that nothing short of a rigorous theory of translation between a natural language and an intensional logic will do before we can consider the latter as providing the logical forms underlying the expressions of that natural language. It was for this reason that he constructed his second intensional logic, which, as we have said, is really a type-theoretical form of set theory with an adjoined theory of senses. The latter is described in terms of two new operators ${ }^{\wedge}$ and ${ }^{\vee}$, called the sense and denotation (or intension-forming and extension-forming) operators, respectively.

We should keep in mind in this context that natural languages are not to be distinguished from artificial or constructed languages on the grounds that only the latter have recursively constructed syntactic categories. After all, a set-theoretic semantics that bypasses the logical forms of intensional logic is possible for a natural language only by providing grammatical rules that allow us to recursively construct the syntactic categories of that language. Indeed, in [UG], Montague formulated a universal grammar whose purpose was precisely "to comprehend the syntax and semantics of both kinds of language within a single natural mathematically precise theory" (p. 222). As part of this universal grammar, Montague also formulated a mathematically precise theory of translation, and as an example of an application of this theory, he constructed a specific translation function that assigned logical forms from his second or sense-denotation intensional logic to the expressions of the fragment of English he formulated within his universal grammar. That is, Montague showed how one can translate a recursively specified natural language into his sense-denotation intensional logic in no less precise a 
manner than one can translate that language into set theory by means of recursive truth-conditions. In this way, Montague overcame the objection that one must rely on circumlocution and linguistic intuition in order to associate logical forms with the expressions of a natural language. Of course, given a theory of logical form that is proposed as an alternative to Montague's sense-denotation intensional logic, the objection remains in force unless a rigorously defined translation function can be defined for that theory as well.

\section{The Incompleteness of Intensional Logic When Based on Membership}

According to Montague, the point to a rigorously characterized translation into intensional logic is that it induces a more perspicuous interpretation of the expressions of natural language than is possible with a purely settheoretic semantics (see [UG], p. 241). Apparently, this is because the logical forms of intensional logic give a representation of intensional entities that is both direct and independent of any antecedently given reference to possible individuals or possible worlds. That, of course, is a claim that is fully consonant with the view of logic as language, and at this point one might even maintain that Montague's sense-denotation intensional logic is an embodiment of the idea of logic as language. The problem with such a conclusion, however, is that the logic is essentially incomplete, and this is not unrelated to the fact that it is based on membership and not on predication.

We must be cautious here in how we are to understand Montague's settheoretic semantics for his intensional logic. For Montague's real commitment to possible individuals and possible worlds is not in his set-theoretic semantics but in the intensional logic that he took to transcend set theory. This intensional logic is really a type-theoretical form of set theory combined with a theory of senses. Every entity of whatever type, for example, is taken in this theory as the denotation (or extension) of a sense (or intension), and properties and relations-in-intension are identified as the senses of sets and relations-in-extension (as represented by their characteristic functions). Propositions are similarly identified as the senses of truth-values. Of course, as objects in their own right (or of their own type), senses are themselves the denotations of other senses, and consequently there is a whole hierarchy of senses in this framework as well. 
In this logic possible individuals are simply the objects indicated by bound occurrences of the individual variables; and that some of these individuals might not actually exist is seen in the fact that $(\forall x)^{\vee} E !(x)$ is not taken by Montague as valid, where $E$ ! stands for the property (or sense) whose denotation is the set of individuals that exists in the actual world. (The set that is the denotation of this property is represented by ${ }^{\vee} E$ !, i.e., by applying the extension-forming operator to E!.) Montague's commitment to possible worlds, on the other hand, is really a commitment to what might be called "world-propositions". For example, where $P, Q$ are propositional variables, the property of being a possible world can be defined as the sense of the set of those propositions $P$ that can denote truth and such that for any proposition $Q$, either $P$ entails $Q$ or $P$ entails the complement of $Q$ :

$$
\text { Poss }-W l d={ }_{d f}^{\wedge}\left[\lambda P\left(\diamond^{\vee} P \wedge(\forall Q)\left(\square\left[{ }^{\vee} P \rightarrow{ }^{\vee} Q\right] \vee \square\left[{ }^{\vee} P \rightarrow{ }^{\vee} \neg Q\right]\right)\right)\right] .
$$

Using this notion, Montague's commitment to possible worlds can now be seen in the fact that

$$
\square(\exists P)\left({ }^{\vee} \text { Poss }-W l d(P) \wedge{ }^{\vee} P\right)
$$

is a valid thesis of his intensional logic (see Prior and Fine 1977, for an analysis of possible worlds as world-propositions).

Now the validity of the above formula is easily seen by returning to Montague's set-theoretic semantics where relative to certain sets that we are to "think of" as the sets of possible individuals and possible worlds, respectively, senses of entities of a given type are represented by arbitrary functions from the set of possible worlds to entities of that type. Propositions are then represented by functions from possible worlds to truth-values (represented in turn by the sets 0 and 1), or equivalently by arbitrary sets of possible worlds, including all unit or singleton sets of possible worlds (or functions that are true at one and only one possible world). A proposition that is represented by such a unit set is then a "world-proposition" in the above sense.

Note that we speak here of propositions being represented by functions from possible worlds (designated or otherwise) to truth values, and not as literally being such functions. This is because for an intensionalist possible worlds are to be analyzed in terms of propositions and not propositions in terms of possible worlds; and for an advocate of Montague's sense-denotation intensional logic in particular, possible worlds are world-propositions in the 
above sense. For such an intensionalist, in other words, functions from possible worlds to truth values can at best be correlated with propositions, not identified with them.

Of course, such a correlation would still imply that propositions are identical when they necessarily denote the same truth values, and that result is problematic. This need not affect the argument for the validity of the above formula, however, so long as an intensionalist can assume that there is always at least one (even if not exactly one) proposition corresponding to each function from possible worlds to truth values. Montague, of course, made the stronger, more problematic assumption of a one-to-one correlation, and, indeed, it appears to be this assumption that is the basis of his claim that we can use set theory to "justify" intensional logic as a framework that transcends set theory itself.

Although Montague assumed that both of his intensional logics transcended set theory, it is really only his sense-denotation intensional logic that contains a type-theoretical form of set theory in its own right. Montague's first intensional logic, as we have said, is really based on predication, and it is only by interpreting predication in terms of membership that Montague was able to think of this logic as containing a type-theoretical form of set theory as well. But an alternative interpretation in which predication is viewed as more fundamental than membership is also possible, and though the logic will contain a theory of classes under that interpretation, these classes will not be sets in the sense of the iterative concept. Indeed, they will instead be classes in the logical sense of the view of logic as language.

Our present concern, however, is with Montague's second or sense-denotation intensional logic, one containing a type-theoretical form of set theory in its own right. This means that a certain necessary condition is imposed on the set-theoretical semantics for this logic; in particular, that the hierarchy of sets (or of their characteristic functions) that is part of this semantics is determined by all finite stages of the operation: $\mathcal{X} \rightarrow \mathcal{P}(\mathcal{X})$, where $\mathcal{P}(\mathcal{X})$ is the power set of $\mathcal{X}$. Or, in terms of functions, and where $a$ and $b$ are arbitrary types of the logic, it is the hierarchy determined by the operation that goes from the universes $\mathcal{U}_{a}, \mathcal{U}_{b}$ of entities of types $a$ and $b$, respectively (and as based on given sets of possible individuals and possible worlds), to the set $\left(\mathcal{U}_{b}\right)^{\mathcal{U}_{a}}$ consisting of all functions from $\mathcal{U}_{a}$ into $\mathcal{U}_{b}$. This is the noncumulative hierarchy of all finite stages generated by the iterative concept of set, and as such it is the basis of what are called the standard models or interpretations of higher-order logic; but standard, it should be emphasized, only 
insofar as such a logic is understood as containing a type-theoretical form of set theory. It is well known that when validity is defined in terms of these standard models, i.e., as truth in all standard models, the set of formulas that are thereby determined to be valid is not recursively enumerable. Any higher-order logic, in other words, that is to be interpreted as containing a type-theoretical form of the iterative concept of set, such as Montague's sense-denotation intensional logic in particular, is essentially incomplete.

A logic that is essentially incomplete cannot be taken as an embodiment of the view of logic as language, or what we have also called the axiomatic approach; or at least it cannot if the set-theoretic semantics with respect to which it is incomplete provides an adequate external criterion of validity for that logic. For in that case, the set-theoretic characterization of validity cannot be eliminated in favor of an axiomatic characterization; and therefore the set-theoretic characterization must be seen as providing more than a guide to validity. This is true, moreover, even when the logic is intended to be interpreted as transcending set theory, as is the case with Montague's sensedenotation intensional logic. In other words, the set-theoretic semantics used to "justify" this intensional logic in fact provides an essential characterization of validity for it; for it is a characterization that cannot be eliminated by any internal criterion in terms of the so-called laws of that logic. Montague's set-theoretic "justification" of his intensional logic does depend on the problematic assumption that propositions can be adequately represented by functions from possible worlds to truth values, but even if this assumption were weakened as indicated above, the logic would still be incomplete, since it still must be interpreted as containing a type-theoretical form of set theory.

A completeness theorem would be forthcoming, it might be noted, if we were to reject the restriction to standard models in the characterization of validity and allow what are called general models as well (see Gallin 1975, chap. 1 , section 3$)$. In that case, the hierarchy of functions need not go from the universes $\mathcal{U}_{a}, \mathcal{U}_{b}$ of entities of types $a$ and $b$, respectively, to the set $\left(\mathcal{U}_{b}\right)^{\mathcal{U}_{a}}$ of all functions from $\mathcal{U}_{a}$ into $\mathcal{U}_{b}$, but only to some nonempty subset of this set that fulfills certain closure conditions. Such an allowance, however, would amount to rejecting the idea that the logic is to contain a type-theoretical form of set theory; for it would amount to replacing the type-theoretical hierarchy of sets by a hierarchy of classes (or of functions) that is based on a concept other than the iterative concept of set. This latter hierarchy is not an implausible framework for an intensional logic based on predication, or at least so we shall subsequently argue, but it is inappropriate for a logic based 
on membership. Redefining validity in terms of general models, in other words, is not a viable alternative for Montague's sense-denotation intensional logic.

\section{Predication Versus Membership in Type Theory}

Both of Montague's intensional logics are theories of logical forms based on one or another version of type theory. This is understandable for Montague's first intensional logic, we maintain, because this logic is based on predication, but it is not really clear why his second, or sense-denotation, intensional logic, which is based on membership, should also be restricted to a type-theoretical theory of logical form. The historical answer as to why one would adopt a type-theoretical theory of logical form is of course avoidance of Russell's paradox. But in the case of Montague's sense-denotation intensional logic, this answer is somewhat problematic.

Russell's paradox really has two forms, one in regard to the class of all classes that are not members of themselves, the other in regard to the property of being a property that is not a property of itself. Russell himself avoided the first form of his paradox by adopting his famous "no classes" theory, according to which all talk of classes is reducible to talk about properties that have those classes as their extensions. He then avoided the second form by imposing type-theoretical restrictions that made it meaningless on grammatical grounds alone to talk about properties being, or not being, properties of themselves. Montague followed Russell in adopting this sort of solution, though the type-theoretical restrictions for his sense-denotation intensional logic are based on Alonzo Church's theory of simple types (supplemented with a hierarchy of types for senses), rather than on Russell's theory of ramified types. Where Montague does not follow Russell in his development of this logic, however, is in not adopting the "no classes" theory. Instead, Montague applies type-theoretical restrictions to talk about classes (or really sets as represented by their characteristic functions) as entities in their own right, as well as to talk about properties and relations in intension as senses of classes and relations in extension.

Now what is problematic about this is that the classes in Montague's intensional logic are really sets or classes in the mathematical sense; i.e., 
they are classes that are formed in accordance with the iterative concept of set. This means that they are formed in accordance with the limitationof-size doctrine according to which sets are not to get too big too fast. In this regard, Russell's paradox is really inapplicable to sets since it assumes a pattern of set-formation that violates the limitation-of-size doctrine and therefore is not in accord with the iterative concept. Russell's paradox in its first form, in other words, is really a paradox about classes in the logical sense, and not about classes in the mathematical sense; that is, it is a paradox about classes as the extensions of properties or concepts, where the latter are based on predication and not on membership. This is why Russell found it natural to avoid this form of his paradox by adopting his "no classes" theory.

Note that unlike sets or classes in the mathematical sense, which have their being in their members, classes in the logical sense have their being in the properties or concepts whose extensions they are. This difference in ontological grounding is not vacuous, moreover, but is based on the difference between membership and predication, or rather on which of these two notions is taken as fundamental. In Montague's intensional logic, for example, membership in a set (as represented by its characteristic function) is fundamental, and the possession of a property, or monadic predication, is analyzed as membership in the set denoted by that property. For example, where $\delta$ is a sense whose denotation is a set of entities of a given type, and $\alpha$ is an entity of that type, then the possession by $\alpha$ of the "property" $\delta$ is defined by Montague as follows (see [UG], p. 236 and [PTQ], p. 259):

$$
\delta\{a\}={ }_{d f}\left[{ }^{\vee} \delta\right](\alpha),
$$

where $\left.{ }^{\vee} \delta\right](\alpha)$ says that the characteristic function of the set denoted by $\delta$ assigns 1 to $\alpha$, i.e., that $\alpha$ belongs to that set. Accordingly, because a similar analysis is given for relational predication as well, it follows that predication, whether monadic or relational, is not a fundamental logical form in Montague's sense-denotation intensional logic but is defined in terms of membership in a set.

Because membership in a set (as represented by the characteristic function of that set), and not predication, is what is really fundamental in Montague's intensional logic, then his way of avoiding Russell's paradox of predication is really a variant of his way of avoiding Russell's paradox of membership. But the latter, we have said, applies only to membership in a class in the logical sense and is inapplicable to sets. Russell's paradox in either form, in other 
words, is irrelevant to the kind of framework Montague has in mind in his sense-denotation intensional logic. But then this leaves us without any real motivation for adopting the type-theoretical restrictions Montague imposed on this logic. ${ }^{1}$

Montague's first intensional logic, on the other hand, is a logic in which predication, and not membership, is fundamental; and in this case, once nominalized predicates are allowed to occur as abstract singular terms, Russell's paradox is not irrelevant. Imposing type-theoretical restrictions on the forms of predication in this logic, accordingly, is an understandable ploy as a way of avoiding Russell's paradox. It is noteworthy, moreover, that instead of rejecting Russell's "no classes" theory in his development of this logic, Montague actually formulated an alternative version of such a theory. A class, for example, can be identified in this logic with a property that has the same extension in every possible world, or what might be called a "rigid" property; and an $n$-ary relation in extension can be similarly identified with an $n$-ary rigid relation in intension. Rigidity in general (i.e., for each natural number n) can be defined as follows (see Montague 1974, p. 132):

$$
\text { Rigid }=_{d f}\left[\lambda F\left(\forall y_{1}\right) \ldots\left(\forall y_{n}\right)\left(\square F\left(y_{1}, \ldots, y_{n}\right) \vee \square \neg F\left(y_{1}, \ldots, y_{n}\right)\right)\right] .
$$

On this analysis, accordingly, an $n$-ary relation-in-extension is simply a rigid relation-in-intension, and a class in the logical sense is simply a rigid, property. This notion of a class should not be confused with the mathematical notion of a set.

The law of logic that we need on this analysis in order to account for our talk of the extension of an arbitrary property or relation in intension is the

\footnotetext{
${ }^{1}$ Without type-restrictions, Montague's sense-denotation intensional logic would amount in effect to a first-order set theory supplemented with a theory of senses in the form of the sense- and denotation-forming operators ${ }^{\wedge}$ and ${ }^{\vee}$, respectively. That is, it would then amount to an applied first-order theory with $\in$ and ${ }^{\wedge}$ and $\vee$ as primitive nonlogical constants. That might actually be preferable to Montague's own description of $\wedge$ and $\vee$ as logical constants; for unlike quantifiers and sentential connectives, these operators have no counterparts in natural language, and the distinctions they are used to articulate are more in the order of an applied theory of logical form than as fundamental constituents of logical forms themselves. This is especially true of the hierarchy of senses that is represented by iterated applications of $\wedge$. For what is represented in this hierarchy is really not in the logical forms of the expressions of natural language, but is rather an interpretation of the occurrence of these expressions in indirect discourse. On Montague's theory, it is, after all, the same word or expression of natural language that is assigned not only a direct sense but an indirect sense as well, and an indirect-indirect sense, and so on ad infinitum.
} 
following principle of rigidity (or what Gallin (1975, p. 77) calls the principle of extensional comprehension):

(PR) $(\forall F)(\exists G)\left(\operatorname{Rigid}_{n}(G) \wedge\left(\forall x_{1}\right) \ldots\left(\forall x_{n}\right)\left[F\left(x_{1}, \ldots, x_{n}\right) \leftrightarrow G\left(x_{1}, \ldots, x_{n}\right)\right]\right)$.

Every property or relation-in-intension, in other words, is co-extensive with a rigid property or relation-in-intension, and all talk of the extension of the former can be analyzed as talk about the latter. Membership in a class that is the extension of a given property, for example, is now analyzed in terms of predication as the possession of a rigid property that is co-extensive with that property. Thus, instead of predication being defined in terms of membership in a set (as represented by the characteristic function of that set), the way it is in Montague's sense-denotation intensional logic, membership in a class is defined in Montague's higher-order modal predicate logic in terms of predication.

One of the noteworthy consequences of (PR) is the commitment to possible worlds in the sense of world-propositions. That is, with (PR) as a basic law of logic,

$$
\square(\exists P)(P \wedge(\forall Q)[\square(P \rightarrow Q) \vee \square(P \rightarrow \neg Q)])
$$

is provable in higher-order modal predicate logic (see my 1986b, Section 11). Given the above analysis of classes in the logical sense, we do not need to rely on a set-theoretic semantics of so-called standard models as a guide to the validity of the above thesis regarding the intensional existence of possible worlds, i.e., the existence of possible worlds as world-propositions.

We should note here, however, that even though predication is fundamental in this logic, and membership is not, Montague himself follows the logic as calculus view and interprets predication in terms of membership in his set-theoretic semantics for this logic. In this way, standard models enter the picture once again; indeed, with respect to such models, the above principle of rigidity, (PR), is easily seen to be valid. But then, of course, with validity defined in terms of standard models we obtain an incompleteness theorem for this logic as well, in which case it too must fail as a candidate for the view of logic as language. That is, insofar as the set-theoretic semantics of standard models provides an adequate external critierion of validity for this logic, then the fact that the logic is incomplete with respect to this semantics shows that the latter provides more than merely a guide to validity; i.e., it shows that the characterization of validity that it does provide cannot be 
eliminated in favor of an internal criterion in terms of the so-called laws of logic.

The weak point in this argument for the view of logic as calculus is the assumption that the standard-model set-theoretic interpretation of predication in terms of membership provides an adequate external criterion of validity for this logic. For it is this interpretation that begs the question as to which is fundamental in this logic, predication or membership. In particular, by imposing an interpretation based on standard models, the classes in the logical sense that are definable within the logic in terms of predication are reinterpreted in effect as sets (or rather as constant functions on possible worlds having these sets as their constant values). That is, the notion of a class in the logical sense is simply discarded on this interpretation in favor of the iterative concept of set.

An alternative, of course, is the replacement of standard models in the set-theoretic characterization of validity by general models, and in particular by general models in which the cardinality of the values of the higher-order predicate variables is no greater than that of the values of the first-order predicate variables. The latter restriction, needless to say, amounts to the assumption that there are no more properties and relations in intension of order $n+1$ than there are of order $n$, for each positive integer $n$. The contrary assumption that there must always be more properties and relations in intension of order $n+1$ than there are of order $n$ is of course based on Cantor's theorem, which is the central feature of the iterative concept of set; and therefore this assumes that the classes of properties and relations in intension of order $n+1$ must all be sets or classes in the mathematical sense of the iterative concept. But this simply begs the question at issue. For when the classes in question are classes in the logical sense, and in particular when they are merely rigid properties and relations-in-intension, then there is no reason why their cardinalities must be in accord with the iterative concept of set. Thus, once we reject the idea that classes in the logical sense are actually sets or classes in the mathematical sense, then there is no reason why there should be more second-order properties than there are first-order properties, or more third-order properties than second-order properties, etc. The typetheoretical division of properties and relations in intension, as a division designed to avoid Russell's paradox, only divides properties and relations-inintension into different types - not into types of different cardinalities. ${ }^{2}$

\footnotetext{
${ }^{2}$ It is noteworthy that Russell was led to the theory of types by considering Frege's
} 
A completeness theorem is forthcoming with respect to this alternative characterization of validity, and there ís no need to think of the set-theoretic semantics of the general models in question as anything more than a guide to validity. Strictly speaking, at least from the standpoint of the view of logic as language, the guidance in this case is really in the opposite direction. For, in order to give the set-theoretic characterization of validity in question, we actually need to rely on certain internal criteria (such as the status of (PR) and the comprehension principle as schematically described basic laws of logic) as constraints that must be imposed on the so-called nonstandard models in question. In other words, what makes the set-theoretic characterization of validity adequate as an external criterion in this case is its dependence on certain basic laws of logic from the internal point of view of logic as language. Of course, given the completeness theorem, the settheoretic characterization of validity can be by-passed altogether in favor of an axiomatic characterization.

Montague's first intensional logic can serve, accordingly, as a candidate for the view of logic as language, at least as far as the problem of a complete axiomatization is concerned. There are other problems, however, including in particular the objection that this sort of logic requires the use of circumlocution or paraphrase in its analysis of the expressions of natural language. But this objection can be overcome once we are given a precise translation function that can be used as the basis of such an analysis. A strategy that suggests itself here for obtaining such a translation for Montague's recursively specified fragment of English is to use the product of two translation functions, one translating Montague's sense-denotation intensional logic into higher-order modal predicate logic, and the other translating the. fragment of English in question into the sense-denotation intensional logic. That is, given Montague's precise specification of the latter translation function, all we need is a formal translation of Montague's sense-denotation intensional logic into his higher-order modal predicate logic. Such a formal translation function has in fact been described by Gallin (1975, Section 13), which means

hierarchy of unsaturated concepts (see my 1986b, Section 8). In this hierarchy (as I have explained in my 1985, Section 2 and 1986b, Sections 4-5), the concepts of any given higher level can be mapped into the concepts of the preceding level, just as first-level concepts can be mapped one-to-one with certain saturated objects called concept-correlates (or Frege's Wertverläufe, given his commitment to extensionality). There is no difference in cardinality, between the concepts of any one level and those of any other level of Frege's hierarchy. 
that we can put aside this objection to Montague's first intensional logic as a version of the view of logic as language.

There is another objection, however, that applies not only to Montague's higher-order modal predicate logic, but to any theory of logical form that is both based on type theory (of third- and higher-order) and proposed as a version of the view of logic as language. In particular, as a framework for conceptual analyses, such a theory imposes inappropriate restrictions on the meaningful use of predicates in natural language. In general, for example, it is meaningless in a theory of types for a nominalized predicate expression to occur in the subject or argument position of another predicate unless the latter can be assigned a higher type than the former; and therefore it is meaningless for any predicate to occur in a nominalized form in its own subject position. Thus, the otherwise unproblematic sentence of English, 'The property of being a property is a property of itself', is by fiat ruled out as meaningless, as is the sentence, 'Smith does not think that the property of being philosophically interesting is itself philosophically interesting, even if Jones does'. Also ruled out in this way are otherwise unproblematic sentences whose predicates apply to nominal expressions of different types, such as, 'Jones thinks that some people are philosophically interesting, as well as that some propositions, properties and relations-in-intension are too'.

The rejection of these and many other meaningful sentences of English is clearly a defect of any theory of logical form proposed as representing the view of logic as language. It is one thing to divide up properties and relationsin-intension as a way of avoiding Russell's paradox of predication, and quite another to make the meaningful use of predicates in natural language actually depend on such a division. Fortunately, there is a way of achieving the one result without also imposing the other, and it is noteworthy that this way involves returning to the sort of framework that Frege and Russell advocated at the turn of the century.

\section{Second-Order Predicate Logic with Nominalized Predicates}

At the turn of the century, both Frege and Russell advocated the idea of logic as language in a form that was in many respects very similar. Both maintained that logic consisted of what today is called "standard" second 
order predicate logic, but supplemented to include a formal account of nominalized predicates as well (see my 1986 b paper for a defense of this claim). ${ }^{3}$ Of course, at that time only Frege had actually constructed a theory of logical form, and his formal account of nominalized predicates was given in his theory of value-ranges (Werwerläufe), or what he also called "classes" in the case of monadic predicates. This was because, unlike Russell, Frege was an extensionalist, not an intensionalist. That is, Frege's universals "differ only so far as their extensions are different" (Posthumous Writings, p. 118), whereas for Russell two universals could have the same extension (see my 1986b, Sections 11 and 13).

Frege's theory of logical form is sometimes described as a second-order set theory; but such a view is quite erroneous. For that view confuses sets or classes in the mathematical sense of the iterative concept with classes in the logical sense. That is, whereas a set, on the iterative concept, has its being in its members, a class as the extension of a concept, according to Frege, "simply has its being in the concept, not in the objects which belong to it" ([1979], p. 183). For Frege the logical forms of predication are more fundamental than that of membership, and this is reflected in the fact that the latter is to be analyzed in terms of the former.

A more appropriate description of Frege's theory is that it is a secondorder predicate logic with nominalized predicates. Originally, in his Begrifsschrift, Frege formulated as an axiom system essentially what today is called "standard" second order predicate logic (with identity). It is clear that he took this logic as providing a logical analysis of the forms of predication that occur in natural language. Later, in his Grundgesetze, Frege added to this logic his theory of value-ranges. This addition was not given as an application of his earlier theory of logical form, it should be emphasized, but as a further development of that theory. In particular, as I have argued in my 1986b article, Sections 4-5, the singular terms generated from formulas by application of the smooth-breathing abstraction operator were interpreted by Frege as symbolic counterparts of the abstract singular terms or noun phrases generated in natural language by predicate nominalizations. These nominalizations include not only such familiar patterns as ' $F$-ness', ' $F$-ity', ' $F$-hood' and 'being an $F$ ', but also infinitives, 'to $F$ ', and gerunds, ' $F$-ing',

\footnotetext{
${ }^{3}$ By standard second-order predicate logic, we do not mean "standard" as interpreted with respect to so-called "standard" models of set theory. Rather, we mean the secondorder predicate that extends standard first-order predicate logic by including a logic for predicate quantifiers that validates the full, impredicative comprehension principle.
} 
as well as Frege's own favorite, 'the concept $F$ '. In other words, Frege viewed the theory of logical forms he developed in the Grundgesetze as providing a logical analysis not only of the predicate expressions that occur in natural language, but also of the predicate nominalizations that occur therein as well.

Russell's conception of logic at the turn of the century was also essentially that of a second-order predicate logic with nominalized predicates, though unlike Frege his account of this logic was presented very informally. (See my 1980 and 1986b articles for a description of Russell's early views.) Russell differed from Frege, however, not only in assuming an intensional theory of universals, but also in maintaining that nominalized predicates denote as singular terms the same entities that predicates otherwise stand for in their role as predicates. Russell rejected, in other words, the Fregean view that universals have an unsaturated nature of their own corresponding to the unsaturated nature of predicates in their role as predicates (1986b, Section $1)$.

Now we shall not be concerned here with the details of either Frege's or Russell's early views on the nature of logic, having already covered that ground in my 1980 and 1986b articles. But, we do want to note and emphasize that what is common to both Frege and early Russell is the idea of second-order predicate logic with nominalized predicates as a paradigm of the view of logic as language. Here we have all of the essentials of what constitutes a theory of logical form as an expression of that view; namely, basic forms of predication, propositional connectives, quantifiers that reach into predicate as well as subject positions, and nominalized predicates as abstract singular terms. These all correspond to fundamental aspects of natural language, and to attempt to do without any of them in a theory of logical form would leave those aspects of natural language unexplained. Of course, these are precisely the features that constitute type-theoretical higher-order predicate logic, except that in the latter predicates are grammatically divided into different types, with the constraint on well-formedness that nominalized predicates can occur as subject (or argument) expressions only of predicates of higher types. This suffices as a way of avoiding Russell's paradox, because the grammatical division imposes a logical division as well. But it turns out that the logical point of the theory of types can be made without the grammatical restrictions, and because the latter impose inappropriate constraints on the meaningful use of predicates in natural language, it is much to be desired that we can make the logical point, and avoid Russell's paradox, without also imposing the grammatical restrictions. What is significant is that this 
can be done by returning to the original context out of which type theory arose, namely, second-order predicate logic with nominalized predicates as abstract singular terms. We can return, in other words, to the paradigm of the view of logic as language.

We can briefly describe this paradigm as follows, where, for convenience, we take $\rightarrow, \neg,=, \forall, \square$, and $\lambda$ as primitive logical constants and assume the others to be defined in the usual way. We assume the availability of denumerably many individual variables, and, for each natural number $n$, denumerably many $n$-place predicate variables. (Propositional variables are taken as 0-place predicate variables.) We will use ' $x$ ', ' $y$ ', ' $z$ ', with or without numerical subscripts, to refer to individual variables, and ' $F^{n}$, , $G^{n}$, , $R^{n}$ ' to refer to $n$-place predicate variables. (Usually we drop the superscript when the context makes clear the number of subject positions that go with a predicate variable.) Complex predicates will all be generated from formulas by means of the $\lambda$-operator. Note that although predicates are not themselves singular terms, they can be transformed into such by deletion of the subject (argument) positions that come with them in their role as predicates. Traditionally, this transformation is marked by a deletion of the parentheses (and commas in the case of a relational predicate) that precede and succeed (or separate in the case of commas) the singular terms to which the predicate can be applied. We shall retain this traditional practice here. For example, on the definition that is to follow, $F(x)$ and $R(x, y)$ are formulas in which $F$ and $R$ occur as predicates, but $G(F)$ and $G(R)$ are formulas in which $F$ and $R$ occur as singular terms. In $F(F)$ and $R(F, R)$, of course, $F$ and $R$ occur both as predicates and as singular terms (though no single occurrence can be both as a predicate and as a singular term). As indicated, we shall require predicates to be accompanied by parentheses (and commas) only when they actually occur in a formula as a predicate.

In the definition that is to follow we use 0 to represent the type of a singular term, 1 the type of a formula, and $n+1$, for $n>0$, the type of an $n$-place predicate expression. For each natural number $n$, accordingly, we recursively define the meaningful expressions of type $n$, in symbols $M E_{n}$, as follows:

1. Every individual variable or constant is in $M E_{0}$, and every $n$-place predicate variable or constant is in both $M E_{n+1}$, and $M E_{0}$;

2. if $a, b \in M E_{0}$, then $(a=b) \in M E_{1}$; 
3. if $\pi \in M E_{n+1}$ and $a_{1}, \ldots, a_{n} \in M E_{0}$, then $\pi\left(a_{1}, \ldots, a\right) \in M E_{1}$;

4. if $\varphi \in M E_{1}$, and $x_{1}, \ldots, x_{n}$ are pairwise distinct individual variables, then $\left[\lambda x_{1}, \ldots x_{n} \varphi\right] \in M E_{n+1}$;

5. if $\varphi \in M E_{1}$, then $\neg \varphi \in M E_{1}$;

6. if $\varphi, \psi \in M E_{1}$, then $(\varphi \rightarrow \psi) \in M E_{1}$;

7. if $\varphi \in M E_{1}$, and $a$ is an individual or a predicate variable, then $(\forall a) \varphi \in$ $M E_{1}$;

8. if $\varphi \in M E_{1}$, then $[\lambda \varphi] \in M E_{0}$; and

9. if $n>1$, then $M E_{n} \subseteq M E_{0}$.

Note that by clause (9) every predicate expression (without its accompanying parentheses and commas) is a singular term. This includes 0-place predicate expressions, but not formulas in general. To nominalize a formula, however, we need only apply clause (8). Thus, we may read ' $[\lambda \varphi]$ ' when it occurs as a singular term as 'that $\varphi$ '. Note also that although only individual variables are bound by the $\lambda$-operator, we can define its application to arbitrary variables $a_{1}, \ldots, a_{n}$ as follows:

$$
\left[\lambda a_{1}, \ldots, a_{n} \varphi\right]=_{d f}\left[\lambda x_{1}, \ldots, x_{n}\left(\exists a_{1}\right) \ldots\left(\exists a_{n}\right)\left(x_{1}=a_{1} \wedge \ldots \wedge x_{n}=a_{n} \wedge \varphi\right)\right],
$$

where $x_{1}, \ldots, x_{n}$ do not occur free in $\varphi$.

In regard to the laws of logic as understood in the original context of this paradigm, we need only take the axioms and rules of "standard" secondorder predicate logic, but applied now to all formulas, including those with as well as those without nominalized predicates among their singular terms (1986b, Section 3). ${ }^{4}$ Because we include $\square$ as a logical primitive, we can add to these axioms and rules those of the $\mathbf{S 5}$ modal propositional logic as well. Of course, Russell's paradox is now derivable as a consequence of the following instance of the comprehension principle:

$$
(\exists F)([\lambda x(\exists G)(x=G \wedge \neg G(x)]=F) .
$$

\footnotetext{
${ }^{4}$ See our earlier note on our use of "standard" with respect to second-order predicate logic.
} 
But instead of following Russell and avoiding the paradox by imposing typetheoretical restrictions on the meaningful use of predicates, we can avoid the paradox by simply imposing the lesser grammatical constraint of excluding all $\lambda$-abstracts that are not homogeneously stratified. ${ }^{5}$ (See my $1986 \mathrm{~b}$ article, Section 9, for an explanation of why heterogeneous stratification is not restrictive enough.) This still allows for the meaningfulness of $F(F)$ and $\neg F(F)$, or even of $[\lambda x \varphi]([\lambda x \varphi])$, so long as $[\lambda x \varphi]$ is homogeneously stratified. Note however that since the $\lambda$-abstract involved in Russell's paradox is not homogeneously stratified, it is excluded by this weaker grammatical constraint as not being well-formed. This means that the original comprehension principle,

$$
\left(\exists F^{n}\right)\left(\left[\lambda x_{1}, \ldots x_{n} \varphi\right]=F\right)
$$

where $F$ does not occur free in $\varphi$, is now restricted to what we shall call the homogeneously stratified comprehension principle $\left(\mathbf{H} \mathbf{S C P} \mathbf{P}_{\lambda}^{*}\right)$.

Except for the restriction to homogeneously stratified $\lambda$-abstracts, it should be emphasized, everything in the original context of our paradigmatic secondorder predicate logic with nominalized predicates remains as it was. We call this system $\square \boldsymbol{\lambda} \mathbf{H S T}^{*}$ (or just $\boldsymbol{\lambda} \mathbf{H S} \mathbf{T}^{*}$ if $\square$ is dropped as a logical constant). In my 1986, it is shown that $\square \boldsymbol{\lambda} \mathbf{H S T}^{*}$ is consistent if weak Zermelo set theory is consistent, and also that it is equiconsistent with the theory of simple types.

It is noteworthy that even the restriction to homogeneously stratified $\lambda$ abstracts can be dropped in favor of the original grammar of our paradigm if we modify instead the standard first-order logic that was also part of that paradigm and allow for denotationless singular terms; that is, if we switch to a first-order logic that is free of existential presuppositions. In this way we can retain the original comprehension principle $\left(\mathbf{C P}_{\lambda}^{*}\right)$, including the instance involved in Russell's paradox, but note that all that follows by Russell's

\footnotetext{
${ }^{5} \mathrm{~A}$ formula or $\lambda$-abstract $\varphi$ is homogeneously stratified iff there is an assignment $t$ of natural numbers to the set of terms and predicate expressions occurring in $\varphi$ such that (1) for all terms $a, b$, if $(a=b)$ occurs in $\varphi$, then $t(a)=t(b)$; (2) for all $n>1$, all $n$ place predicate expressions $\pi$ and all terms $a_{1}, \ldots, a_{n}$, if $\pi\left(a_{1}, \ldots, a_{n}\right)$ occurs in $\varphi$, then (i) $t\left(a_{j}\right)=t\left(a_{k}\right)$, for $1 \leq j, k \leq n$, and (ii) $t(\pi)=t\left(a_{1}\right)+1$; (3) for all natural numbers $m$, all individual variables $x_{1}, \ldots, x_{m}$, and all formulas $\psi$, if $\left[\lambda x_{1}, \ldots x_{m} \psi\right]$ occurs in $\varphi$, then (iii) $t\left(x_{j}\right)=t\left(x_{k}\right)$, for $1 \leq j, k \leq m$, and (iv) $t\left(\left[\lambda x_{1}, \ldots x_{m} \psi\right]\right)=t\left(x_{1}\right)+1$; and (4) for all formulas $\chi$ if $[\lambda \chi]$ occurs in $\varphi$, and $a_{1}, \ldots, a_{k}$ are all the terms or predicates occurring in $\chi$, then $t(\chi) \geq \max \left[t\left(a_{1}\right), \ldots, t\left(a_{k}\right)\right]$.
} 
argument is that

$$
\neg(\exists y)([\lambda x(\exists G)(x=G \wedge \neg G(x))]=y)
$$

is now provable. This does not contradict

$$
(\exists F)([\lambda x(\exists G)(x=G \wedge \neg G(x))]=F)
$$

as an instance of $\left(\mathbf{C P}_{\lambda}^{*}\right)$, but only requires that we distinguish the role of $[\lambda x(\exists G)(x=G \wedge \neg G(x))]$ as a predicate from its role as a singular term. Russell's contention that nominalized predicates denote as singular terms the same entities that predicates otherwise stand for in their role as predicates must be rejected, and something like Frege's original contention of a distinction between unsaturated concepts and saturated objects retained (see my 1986b, Section 17, for an interpretation of this distinction different from Frege's). All of the properties and relations denoted by singular terms in $\square \boldsymbol{A H S T}^{*}$ can be retained, however, by adding to our modified context a special axiom $\left(\exists / \mathbf{H S C P}_{\lambda}^{*}\right)$ to that effect $1986 \mathrm{~b}$, Section 15 and 1985, Section 7 ). We call the resulting system $\mathbf{H S T}_{\lambda \square}^{*}$ (or just $\mathbf{H S T}_{\lambda}^{*}$ if $\square$ is dropped as a primitive logical constant). In my 1986 it is shown that $\mathbf{H S T}_{\lambda \square}^{*}$ is equiconsistent with $\square \boldsymbol{\lambda} \mathbf{H S T}^{*}$, and therefore with the theory of simple types as well.

Both $\square \boldsymbol{\lambda} \mathbf{H S T} \mathbf{T}^{*}$ and $\mathbf{H S T}_{\lambda \square}^{*}$, it should be emphasized, are reconstructions of the original context of our paradigm. Both, in their own way, make the logical point of the theory of types, but without also imposing the grammatical restrictions of the latter. This logical point was actually already present in Frege in his hierarchy of unsaturated concepts and was taken over by Russell in his attempt to avoid his paradox (1986b, Section 8). In this regard we are not really changing the original context of our paradigm so much as correcting the way that properties and relations are to be posited in that paradigm.

We should also note here that $\square \boldsymbol{\lambda} \mathbf{H S T}^{*}$ has been used in Montague grammar by Gennaro Chierchia in place of Montague's own sense-denotation intensional logic, and that in fact Chierchia has shown $\square \boldsymbol{\lambda} \mathbf{H S T}^{*}$ to be a better semantical framework over all known alternatives as a way of explaining a variety of issues in linguistics (see Chierchia 1984, 1985). This application of $\square \boldsymbol{A H S T}{ }^{*}$ has involved the construction of a precise translation function from English into $\square \boldsymbol{\lambda} \mathbf{H S} \mathbf{T}^{*}$, and, as a result, the objection that a conceptual analysis of natural language in terms of $\square \boldsymbol{\lambda} \mathbf{H S T}^{*}$ requires the use of circumlocution and paraphrase is without force. Since such a translation will apply 
to $\mathbf{H S T}_{\lambda \square}^{*}$ as well, these results indicate how either system can be taken as a reconstruction of our original paradigmatic view of logic as language.

Finally, let us note that the principle of rigidity, (PR), formulated earlier for higher-order modal predicate logic is also well formed in $\square \boldsymbol{\lambda} \mathbf{H S T}^{*}$ and $\mathbf{H S T}_{\lambda \square}^{*}$. Adding (PR) to either of these systems implies the existence of possible worlds in the sense of world-propositions, just as it does in higher-

order modal predicate logic. But there is another sense of the notion of a possible world now available as well; namely, the notion of a possible world as a maximal "class" of compossible facts. Thus, where facts are defined as true propositions (or as what true propositional forms denote when nominalized), i.e., where

$$
\text { Fact }{ }_{d f}[\lambda x(\exists P)(x=P \wedge P)],
$$

and a class in the logical sense is just a rigid, property, this notion of a possible world can be defined as follows:

$$
\text { Poss-Wld }{ }_{2}{ }_{d f}\left[\lambda x(\exists F)\left(x=F \wedge \operatorname{Rigid}_{1}(F) \wedge \diamond(\forall y)[F(y) \leftrightarrow F a c t(y)]\right)\right] .
$$

Now just as (PR) implies the existence of possible worlds in the sense of world-propositions, so too (PR) in either of our reconstructed systems implies the existence of possible worlds in the sense of maximal "classes" of compossible facts. That is,

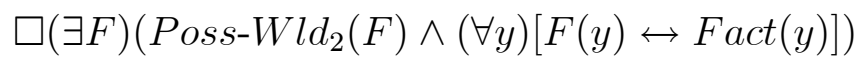

is provable on the basis of (PR) in either of these systems. We should note here that none of these results depends on the problematic view of properties and relations in intension as being identical when necessarily co-extensive. That is, the principle of intensionality,

$$
\square\left(\forall x_{1}\right) . .\left(\forall x_{n}\right)(\varphi \leftrightarrow \psi) \rightarrow\left[\lambda x_{1} \ldots x_{n} \varphi\right]=\left[\lambda x_{1} \ldots x_{n} \psi\right],
$$

need not be taken as a basic law of logic in either of these reconstructions of our original paradigm of the view of logic as language.

\section{A Set-Theoretic Semantics with Predica- tion as Fundamental}

As reconstructions of the original paradigm of the view of logic as language, both $\square \boldsymbol{\lambda} \mathbf{H S T}^{*}$ and $\mathbf{H S T}_{\lambda \square}^{*}$ take predication, and not membership, as fun- 
damental. This is a feature we should retain, accordingly, even in the settheoretic semantics we shall construct for these systems. Indeed, as we will see, with predication given its own special representation within a semantic structure, the distinction between standard and nonstandard models becomes void, which is as it should be since the classes we want to represent in these structures are classes in the logical sense.

One of the notions that we will use in this semantics is the idea of a Fregean correlation between the entities that predicates stand for in their role as predicates and the objects or individuals that are denoted by their nominalizations when they occur as abstract singular terms. We do not preclude the possibility that this correlation is the identity function on the entities in question, however; that is, the semantics is neutral between a Fregean and a Russellian interpretation of nominalized predicates. The semantics is neutral in other respects as well, as we shall see, but these other features will not concern us here. (See my 1978 for a fuller discussion of the issue of a semantics that is neutral between competing versions of logic as language.)

Because the semantics we describe here is a modalized and Fregean modification of John Simms's (1980) semantics, we will refer to the semantic structures in question as modal $S^{*}$-structures. (The present version of this semantics was first given in my (1986, Chap. 6) and another, slightly different, version was given in my (1978, Section 11).) Accordingly, where $\mathfrak{A}=\left\langle D_{n}, E_{n}, H_{i}, f_{i}\right\rangle_{n \in \omega, i \in W}$, we will say that $\mathfrak{A}$ is a modal $S^{*}$-structure if and only if

1. $E_{n} \subseteq D_{n}$, for all $n \in \omega$;

2. $D_{m+1} \cap D_{n+1}=0$, for all $m, n \in \omega$ such that $m \neq n$;

3. for $i \in W, H_{i} \subseteq \cup_{n \in \omega}\left(D_{n+1} \times\left(D_{0}\right)^{n}\right)$;

4. for $i \in W, F_{i}$ is a function from $\cup_{n \in \omega} D_{n}$ into $D_{0}$ such that for all $d \in E_{0}, f_{i}(d)=d$; and

5. for $n \in \omega, W$ and $D_{n}$ are not empty.

By way of explanation, "think of" the members of $W$ as possible worlds and the sets $D_{n}$ and $E_{n}$ as the ranges, respectively, of the free and the bound variables of type $n$. Then, what clause (1) requires is that all values of the bound 
variables of type $n$ are also values of the free variables of type $n$, for all $n \in \omega$. Structures in which $E_{n}$ is a proper subset of $D_{n}$ are free of existential presuppositions regarding expressions of type $n$. However, because in our present context we want the comprehension principle of either $\square \boldsymbol{\lambda} \mathbf{H S T}^{*}$ or $\mathbf{H S T}_{\lambda \square}^{*}$ to be externally valid, we will exclude those structures in which $D_{n} \neq E_{n}$, for $n>0$. Note, however, that in $\mathbf{H S T}_{\lambda \square}^{*}$ singular terms are free of existential presuppositions, and therefore in the structures characterizing validity in $\mathbf{H S T}_{\lambda \square}^{*} E_{0}$ is a proper subset of $D_{0}$. In $\square \boldsymbol{\lambda} \mathbf{H S T}^{*}$, on the other hand, singular terms, including nominalized predicates, are posited as always denoting, and therefore in structures characterizing validity in $\square \boldsymbol{\lambda} \mathbf{H S T}^{*}, E_{0}=D_{0}$. These differences between the structures characterizing set-theoretic validity for $\square \boldsymbol{\lambda} \mathbf{H} \mathbf{S T}^{*}$, as opposed to those characterizing set-theoretic validity for $\mathbf{H S T}_{\lambda \square}^{*}$ are determined by criteria internal to these systems as separate versions of the view of logic as language.

What clause (2) in our definition of a modal $S^{*}$-structure requires is that no $m$-ary universal is an $n$-ary universal if $m \neq n$, and this of course is as it should be. Where $i \in W$, clause (3) describes the set-theoretic relation that we are to "think of" as predication with respect to $i$. Thus, in particular, we are to "think of" the first component of the ordered pairs in this relation as an $n$-ary universal (a member of $D_{n+1}$ ), for some $n \in \omega$, and the second component as an $n$-tuple drawn from the domain of individuals. There is nothing in this description, it should be emphasized, that involves interpreting predication at $i$ as membership; that is, there is no presumption that the relation in question has any similarity at all to membership (of an $n$-tuple) in a set. Of course, in general, relative to the internal criteria of a given theory of logical form as a version of the view of logic as language, including especially the comprehension principle that is internally valid in such a theory, the relation representing predication at a given possible world will have a more determinate structure than is described in clause (3); and because it is determined by the laws of logic of the theory in question, this more determinate structure will remain invariant across all possible worlds, even though the particular relation assigned to predication at one possible world will in general be different from that assigned at another. This latter feature, i.e., that different relations may be assigned to predication at different possible worlds, is a consequence of the essential indexical nature of predication. That predication is essentially indexical, and not "timeless" or "worldless", as is sometimes claimed, explains why the same property can be predicated of different objects at different possible worlds (or at differ- 
ent times in the same possible world), whereas a set, having its being in its members, cannot have different members at different possible worlds (or at different times in the same world).

Finally, where $i \in W$, the function $f_{i}$ is to be our Fregean correlation at $i$ of universals with individual objects. We allow this correlation to be indexical, i.e., to vary at different possible worlds, only in the semantics that is neutral between alternative theories. For example, in a modal counterpart of Frege's theory, a nominalized predicate is interpreted as denoting at a given possible world the class that is the extension at that world of the universal otherwise assigned to that predicate. But since the same universal will in general have different extensions at different possible worlds, it follows that a nominalized predicate may denote different objects at different possible worlds. In other words,

$$
(\exists F)(\exists G)(F=G \wedge \diamond F \neq G)
$$

is consistent in such a modal Fregean framework (1986b, Section 16). In $\square \boldsymbol{\lambda} \mathbf{H S T}^{*}$ and $\mathbf{H S T}_{\lambda \square}^{*}$, however, Leibniz's law is valid in its unrestricted form, and as a consequence, for all $i, j \in W, f_{i}=f_{j}$. A modal $S^{*}$-structure where this holds is said to be rigid. As indicated, set-theoretic validity for $\square \boldsymbol{\lambda} \mathbf{H S T}^{*}$ or $\mathbf{H S T}_{\lambda \square}^{*}$ will be defined only with respect to rigid modal $S^{*}$ structures that satisfy the basic laws of logic of such a system. (Some principles will be valid in all rigid modal $S^{*}$-structures. These are the principles of the minimal system $\square \boldsymbol{\lambda} \mathbf{M}^{*}$ described in my 1986 (Chap. 5 and 1978, Section 10.)

We will forego the details here of the definition of satisfaction in a modal $S^{*}$-structure at a given possible world (1986, Section 6.5 ). We should note, however, that unlike the situation in the usual set-theoretic semantics for second and higher-order predicate logic (where predication is interpreted as membership), this definition in no way depends on either the notion of a standard model that is "full" (in the sense of the iterative concept of set), or of a nonstandard model that, if not "full", is at least closed under conditions that enable us to assign semantic values to complex expressions. Indeed, with predication taken as fundamental in our semantics as well as our syntax, there is no basis at all for the kind of distinction that obtains between standard and nonstandard models when predication is interpreted as membership. For with predication as fundamental, $n$-ary universals are no longer analyzed or represented by functions from possible worlds to sets of $n$-tuples, 
but are rather directly represented in the same way that possible worlds and individuals are. This cuts the ground completely from the kind of incompleteness theorem that is generated with respect to the so-called standard models of higher-order logic; and this is as it should be, for reasons already explained, when it is intended that predication, and not membership, be taken as fundamental in the higher-order logic in question. It also cuts the ground from the idea that properties and relations-in-intension are identical when they have the same extension in every possible world.

Finally, it should be emphasized that except for a certain minimal system of principles that are valid in all (rigid) modal $S^{*}$-structures, a completeness theorem is not forthcoming for a system such as $\square \boldsymbol{\lambda} \mathbf{H S T}{ }^{*}$ or $\mathbf{H S T}_{\lambda \square}^{*}$ except by restricting the $S^{*}$-structures to those in which the basic laws of the system are valid. (A formula is valid in an $S^{*}$-structure if it is true in all possible worlds of that structure.) This is not because these systems are incomplete otherwise, but rather because it is only by excluding certain $S^{*}$-structures that we can give a set-theoretical (or really mathematical) representation of the additional content of the basic laws of logic that are valid in these systems over and above those of the minimal system. In other words, on the view of logic as language, where predication and not membership is fundamental, the set-theoretical definition of validity for a system such as $\square \boldsymbol{\lambda} \mathbf{H S T}^{*}$ or $\mathbf{H S T}_{\lambda \square}^{*}$ provides a strictly external and extrinsic criterion that in no way is essential to the notion of validity that is internal to this system. The real content of our logic, on this view, is not our set theory, but in the logic itself as a formal theory of predication.

\section{Concluding Remarks}

The account we have given here of the view of logic as language should not be taken as a rejection of the set-theoretical approach or as defense of the metaphysics of possibilist logical realism. Rather, our view is that there are really two types of conceptual framework corresponding to our two doctrines of the nature of logic. The first type of framework is based on membership in the sense of the iterative concept of set; and although extensionality is its most natural context (because sets have their being in their members), it may nevertheless be extended to include intensional contexts by way of a theory

of senses (as in Montague's sense-denotation intensional logic). The second type of framework is based on predication; and in certain developments it 
can be associated with one or another theory of universals. Extensionality is not the most natural context in this theory, but where it does hold and extensions are posited, the extensions are classes in the logical and not in the mathematical sense.

Russell's paradox, as we have explained, has no real bearing on setformation in a theory of membership based on the iterative concept of set, but it does bear directly on concept-formation or the positing or universals in a theory based on predication. As a result, our second type of framework has usually-been thought to be incoherent or philosophically bankrupt, leaving us with the set-theoretical approach as the only viable alternative. That is why so much of analytic philosophy in the 20th Century has been dominated by the set-theoretical approach. Set theory, after all, does seem to serve the purposes of a mathesis universalis.

What is adequate as a mathesis universalis, however, need not therefore also be adequate as a lingua philosophica or characteristica universalis. In particular, the set-theoretic approach does not seem to provide a philosophically satisfying semantics for natural language; this is because it is predication and not membership that is fundamental to natural language. An adequate semantics for natural language, in other words, seems to demand a conceptual framework based on predication and not on membership.

It has been our contention here that Russell's paradox has not really nullified the second type of conceptual framework, i.e., one based on predication. And in fact we have shown that one can return to Frege's and Russell's original paradigm of second-order predicate logic with nominalized predicates as a coherent and philosophically useful theory of predication. Being based on predication, this framework is not subject to the incompleteness problem the way a theory of membership is. In addition, we do not need to resort to circumlocution or paraphrase in the application of this framework to natural language. To be sure, we have described this framework, and motivated our discussion throughout, primarily in terms of logical realism as its associated theory of universals, i.e., with propositions, properties and relations in intension as the basic entities involved in predication, and with possible worlds as either certain kinds of propositions or certain kinds of properties. We have done so because this ontology, together with a commitment to possible individuals, seems to be implicit in natural language. But we do not mean to claim that this is the only theory of universals, or that $\square \boldsymbol{A H S T} \mathbf{H}^{*}$ and $\mathbf{H S T}_{\lambda \square}^{*}$ with or without (PR), the principle of rigidity, are the only formal theories of predication that can provide an adequate semantics for natural 
language. In other words, there are other theories of universals, as well as other formal theories of predication, and only a comparison of their strengths and weaknesses will help us to decide which we should adopt as a framework for natural language. (It is this sort of study that I have initiated in my (1986) book and my 1988 article.)

We do not maintain, accordingly, that we should give up the set-theoretic approach, especially when dealing with the philosophy and foundations of mathematics, or that only a theory of predication associated with possibilist logical realism will provide an adequate semantics for natural language. In both cases we may find a principle of tolerance, if not outright pluralism, the more appropriate attitude to take.

\section{References}

[1] Chierchia, Gennaro: 1984, Topics in the Syntax and Semantics of Infinitives and Gerunds, Ph.D. Dissertation, University of Massachusetts, Amherst.

[2] Chierchia, Gennaro: 1985, 'Formal Semantics and the Grammar of Predication', Linguistic Inquiry 16, 417-43.

[3] Cocchiarella, Nino B.: 1986, Logical Investigations of Predication Theory and the Problem of Universals, vol. 2 of Indices, Bibliopolis Press, Naples.

[4] Cocchiarella, Nino B.: 1978, 'On the Logic of Nominalized Predicates and its Philosophical Interpretations', Erkenntnis 13, 339-69; corrigendum in vol. 14, pp. 103-04.

[5] Cocchiarella, Nino B.: 1980, 'The Development of the Theory of Logical Types and the Notion of a Logical Subject in Russell's Early Philosophy', Synthese 45, 71-115.

[6] Cocchiarella, Nino B.: 1985, 'Frege's Double Correlation Thesis and Quine's Set Theories NF and ML', Journal of Philosophical Logic 14, $1-39$.

[7] Cocchiarella, Nino B.: 1986b, 'Frege, Russell and Logicism: A Logical Reconstruction', in Haaparanta Leila and Jaakko Hintikka (eds.), Frege Synthesized, D. Reidel, Dordrecht, 197-252. 
[8] Cocchiarella, Nino B.: 1988, 'Philosophical Perspectives on Formal Theories of Predication', in Handbook of Philosophical Logic, vol. IV, Gabbay, Dov and Franz Guenthner (eds.), D. Reidel, Dordrecht.

[9] Frege, Gottlob: 1979, Posthumous Writings, H. Hermes, F. Kambartel, and F. Kaulbach, eds., Translated by P. Long and R. White, The University of Chicago Press, Chicago.

[10] Gallin, Daniel: 1975, Intensional and Higher-Order Modal Logic, NorthHolland, Amsterdam.

[11] van Heijenoort, Jean: 1967, 'Logic as Language and Logic as Calculus', Synthese 17, 324-30.

[12] Hintikka, Jaakko: 1980, 'Theories of Truth and Learnable Languages', in S. Kanger and S. Ohman (eds.), Philosophy and Grammar, D. Reidel, Dordrecht, pp. 37-57.

[13] Hintikka, Jaakko: 'Semantics: A Revolt Against Frege', in G. Floistad (ed.), Contemporary Philosophy: A New Survey, Martinus Nijhoff, The Hague, pp. 57-82.

[14] Montague, Richard: 1974, Formal Philosophy, Selected Papers of Richard Montague, edited and introduction by R. H. Thomason, Yale University Press, New Haven. The papers referred to in this volume are the following:

[EFL]: 'English as a Formal Language'.

$[\mathrm{PE}]:$ 'On the Nature of Certain Philosophical Entities'.

[PTQ]: 'The Proper Treatment of Quantification in Ordinary English'. [UG]: 'Universal Grammar'.

[15] Moravcsik, J.: 1979, 'Grammar and Meaning', in I. Niiniluoto and M. B. Provence Hintikka (eds.), Essays in Honour of Jaakko Hintikka, D. Reidel, Dordrecht, pp. 3-15.

[16] Prior, Arthur and Kit Fine: 1977, Worlds, Selves, and Times, Duckworth Press, London.

[17] Quine, W. V. O.: 1968, 'Replies', in Essays in Honor of W. V. Quine, Synthese 19. 
[18] Simms, John: 1980, 'A Realist Semantics for Cocchiarella's T*', Notre Dame Journal of Formal Logic 21, 1-32. 